\title{
Adjuvante Chemotherapie in der Behandlung der Urothelkarzinome des oberen Harntraktes
}

\author{
Lea Pape · Johanna Richter · Jürgen Dunst ${ }^{1}$ \\ Online publiziert: 19. Juni 2020 \\ (c) Der/die Autor(en) 2020
}

Hintergrund Beim Urothelkarzinom des oberen Harntraktes (UTUC) handelt es sich um eine eher seltene maligne Erkrankung mit einer Inzidenz von 2 pro 100.000 Einwohnern pro Jahr. Die UTUCs machen nur 5-10\% aller Urothelkarzinome aus [1, 2]. Obwohl sowohl das Blasenkarzinom als auch das UTUC aus dem Übergangsepithel entstehen, zeigen sich doch Unterschiede hinsichtlich Genaberrationsmuster, initialdiagnostischem Stadium und Prognose [3-5]. Hinsichtlich der Prognose schneiden UTUCs im Vergleich zu dem Urothelkarzinom der Blase schlechter $\mathrm{ab}$, da sie häufiger in höheren Stadien diagnostiziert werden [6]. Bisher besteht die Standardtherapie für das fortgeschrittene UTUC aus einer radikalen Nephroureterektomie, gefolgt von klinischen und radiologischen Nachbeobachtungen. Fortgeschrittene UTUC zeigen unter dieser Therapie eine schlechte Prognose mit 5-Jahres-Überlebensraten unter $50 \%$ [1]. Bisherige Studien zum ergänzenden Einsatz von Chemotherapie sind vornehmlich retrospektiv und mit geringer statistischer Aussagekraft [7-12]. Aufgrund der geringen Datenlage konnten deshalb bisher keine Empfehlungen zum Einsatz einer perioperativen Chemotherapie beim UTUC gegeben werden [1, 7]. Die in 2020 veröffentlichte POUT-Studie (,Peri-Operative chemotherapy versus sUrveillance in upper Tract urothelial cancer") stellt nun die größte multizentrische, prospektive und randomisierte Phase-3-Studie zur Wirksamkeit einer adjuvanten Chemotherapie nach Nephroureterektomie (NUE) bei Patienten mit UTUC dar.

Originalpublikation Birtle A, Johnson M, Chester J et al (2020) Adjuvant chemotherapy in upper tract urothelial carcinoma (the POUT trial): a phase 3 , open-label, randomised controlled trial. Lancet 6736(20):1-10. https://doi.org/10.1016/S01406736(20)30415-3

Prof. Dr. med. Jürgen Dunst

Juergen.Dunst@uksh.de

1 Klinik für Strahlentherapie, Universitätsklinikum Schleswig-Holstein, Campus Kiel, Feldstr. 21, 24105 Kiel, Deutschland
Patientengut und Methoden Einschlusskriterien für die Studie waren: Metastasenfreiheit (M0), lokal fortgeschrittenes Tumorstadium oder Lymphknotenbefall sowie eine glomeruläre Filtrationsrate (GFR) von mindestens $30 \mathrm{ml} / \mathrm{min}$. Das Therapiekonzept bestand aus einer adjuvanten Chemotherapie mit 4 Zyklen zu jeweils 21 Tagen, die innerhalb von 90 Tagen nach der NUE (plus Exzision klinisch/radiologisch auffälliger Lymphknoten) begonnen wurde. Alle Patienten in der Interventionsgruppe erhielten an den Tagen 1 und 8 Gemcitabin sowie zusätzlich am Tag 1 Cisplatin, sofern die GFR $\geq 50 \mathrm{ml} / \mathrm{min}$ betrug. Bei niedrigerer GFR wurde Cisplatin durch Carboplatin ersetzt. Sowohl die Interventions- als auch die Kontrollgruppe wurden in regelmäßigen Abständen über im Median 30 Monate klinisch und radiologisch kontrolliert, die unerwünschten Nebenwirkungen der Therapie erfasst und ein standardisierter Fragebogen zur Lebensqualität ausgefüllt. Als primärer Endpunkt der Studie wurde das ,disease free survival“ (DFS) festgelegt; Ereignisse waren Rezidive, Metastasen oder Tod. Sekundäre Endpunkte waren das Gesamtüberleben, die Compliance, die kurzfristige und langfristige Toxizität der Behandlung sowie die Lebensqualität.

Ergebnisse Im Zeitraum von Juni 2012 bis November 2017 wurden 261 Patienten an 57 Studienstandorten rekrutiert, wovon in der Randomisierung 132 Patienten der Chemotherapiegruppe und 129 Patienten der Kontrollgruppe zugeordnet wurden. Das mittlere Alter der Patienten betrug 68,5 Jahre. 94\% der Patienten wurden im Stadium pT2/T3 diagnostiziert, $91 \%$ hatten keinen Lymphknotenbefall, wobei bei fehlenden histopathologischen Ergebnissen die Bewertung der Bildgebung ausschlaggebend war. Die GFR der Patienten lag bei $64 \%$ aller Teilnehmer bei $\geq 50 \mathrm{ml} / \mathrm{min}$. Auf Empfehlung des unabhängigen Datenüberwachungskomitees wurde die Patientenrekrutierung vorzeitig beendet, nachdem bereits frühzeitig das Kriterium der Wirksamkeit erreicht worden war. Die bereits erhobenen Daten zeigten innerhalb der Interventionsgruppe weniger krankheitsbezogene Ereignisse (27\% im Vergleich zu $47 \%$ in der Kontrollgruppe). Die adjuvante Chemotherapie reduzierte dabei das 
Risiko für das Auftreten von Rezidiven um $55 \%$ (,hazard ratio" $[\mathrm{HR}]=0,45,95 \%$-Konfidenzintervall $(95 \%$-KI) $0,3-0,68, p=0,0001)$. Im Vergleich der Schätzungen des krankheitsfreien 3-Jahres-Überlebens präsentierte die Chemotherapiegruppe mit $71 \%$ (95\%-KI: 61-78) wesentlich bessere Werte als die Kontrollgruppe mit 46\% (95\%-KI: 11-38). Auch nach einer Angleichung bekannter prognostischer Faktoren blieb der Effekt der Chemotherapie unverändert; er war in allen Prognosegruppen ähnlich. In der Subgruppenanalyse zeigte sich ein signifikanter Effekt nur bei Patienten der Gemcitabin-Cisplatin-Gruppe ( $\mathrm{HR}=0,35$, $p=0,0002)$. In der mit Gemcitabin/Carboplatin behandelten Subgruppe war der Effekt geringer und nicht signifikant $(\mathrm{HR}=0,66, p=0,21)$. Der Einfluss auf das Gesamtüberleben konnte allerdings aufgrund der vorzeitigen Veröffentlichung der Daten noch nicht berechnet werden. Er ist für einen späteren Zeitpunkt geplant. Zum Zeitpunkt der aktuellen Analyse waren in der Chemotherapiegruppe 24 Todesfälle und in der Kontrollgruppe 38 Todesfälle aufgetreten.

Innerhalb der Chemotherapiegruppe traten mit $44 \%$ mehr unerwünschte therapiebedingte Ereignisse (CTCAE $\geq$ Grad 3) auf im Vergleich zu 4\% in der Kontrollgruppe. Dabei handelte es sich um aus der klinischen Praxis bekannte, unerwünschte Ereignisse des jeweiligen Chemotherapieregimes, von denen keines tödlich verlief. In Analysen zur Lebensqualität wurden die Fragebögen von 243 Patienten ausgewertet. Patienten der Chemotherapiegruppe zeigten vorübergehend eine eingeschränkte Lebensqualität während sowie 3 Monate nach der Therapie. Diese glich sich nach 6 Monaten wieder den Werten der Kontrollgruppe an.

Schlussfolgerungen der Autoren Die Autoren empfehlen, das untersuchte Therapiekonzept als neuen Standard festzulegen. Zur Wirksamkeit von Carboplatin als Ersatz für Cisplatin bei herabgesetzter Nierenfunktion konnte noch keine Aussage gemacht werden. Ob aber bei UTUC standardmäßig eine extensive abdominale Lymphknotendissektion durchgeführt werden sollte oder nicht, ist Gegenstand aktueller Diskussionen. Deshalb wurden diesbezüglich zugunsten der Durchführbarkeit in der POUT-Studie keinerlei Vorgaben gemacht, trotz der Gefahr eines ,under staging“ durch Übersehen von Mikrometastasen.

\section{Kommentar}

Die bisherige Literatur zur systemischen Therapie des UTUC hatte zwar bereits auf die Vorteile einer perioperativen Chemotherapie hingewiesen [13], basierte aber vornehmlich auf retrospektiven Studien sowie einzelnen prospektiven Studien mit geringen Fallzahlen [8]. In Studien zur Anwendung der adjuvanten Chemotherapie beim UTUC präsentierten sich uneindeutige Ergebnisse: Wäh- rend einige Studien signifikante Überlebensvorteile einer adjuvanten Therapie beschrieben [10, 14], zeigten andere keinen Effekt auf die Prognose der Patienten [11, 15]. Metaanalysen von nichtrandomisierten Studien zur adjuvanten Chemotherapie beim UTUC deuteten auf signifikante Verbesserungen des Gesamt- sowie des krankheitsfreien Überlebens durch eine adjuvante Chemotherapie hin [8] Außerdem können durch den Einsatz von neoadjuvanten Therapieschemata bei UTUC-Patienten ein signifikantes „downstaging“ und pathohistologische Remissionen erreicht werden $[16,17]$. Einige Studien beschrieben auch signifikante Effekte der platinbasierten neoadjuvanten Chemotherapie auf das Überleben von UTUC-Patienten [12, 18]. In der Summe war die Datenlage aber nicht ausreichend, um eindeutige Empfehlungen zu geben.

Bei der POUT-Studie handelt es sich um die erste prospektive randomisierte Phase-3-Studie zur Anwendung einer perioperativen Chemotherapie beim Urothelkarzinom des oberen Harntraktes [19]. Angesichts des Mangels an hinlänglichen Studien liefert die POUT-Studie damit zum ersten Mal Daten zur adjuvanten Chemotherapie beim UTUC, die aufgrund des Studiendesigns in geringerem Maße von systematischen Fehlern (Bias) betroffen sind. Jedoch müssen bei der Beurteilung der Ergebnisse einige Einschränkungen innerhalb der Studie betrachtet werden:

Zum einen gab es keine klaren Vorgaben zur (erweiterten) Lymphknotendissektion. Ein einheitliches Vorgehen wäre hierbei wünschenswert gewesen, um eine verlässliche Aussage dazu treffen zu können, ob die adjuvante Chemotherapie in der Lage ist, auch bei weiter fortgeschrittenen Erkrankungen und Mikrometastasen eine Verbesserung des krankheitsfreien Überlebens zu erzielen. Die Ergebnisse der Studie konnten dies für Patienten mit Lymphknotenbefall nämlich nicht eindeutig belegen.

Darüber hinaus ist auch der adjuvante Ansatz in der POUT-Studie zu hinterfragen. Es wurde nämlich bislang noch keine randomisierte Studie zur Frage durchgeführt, ob beim UTUC eine adjuvante oder eine neoadjuvante Chemotherapie einen größeren Nutzen aufweist [7]. Zieht man wiederum den Vergleich zum Blasenkarzinom heran, wäre eine neoadjuvante Therapie zu bevorzugen [20]. Wie schon von den Autoren der POUT-Studie selbst angesprochen, brächte die neoadjuvante Chemotherapie einige Vorteile mit sich, sofern es gelänge, die Patienten adäquat zu selektionieren [21].

In den adjuvanten Therapieansatz wurden ausschließlich Patienten mit einer GFR $\geq 30 \mathrm{ml} / \mathrm{min}$ eingeschlossen. Durch eine NUE kommt es aber zu einem signifikanten Abfall der GFR [22]. Legte man den üblichen Grenzwert zur GFR von $60 \mathrm{ml} / \mathrm{min}$ für den Einsatz von Cisplatin zugrunde [19], so könnte es sich zeigen, dass nach dem operationsbedingten Abfall der GFR ca. 30\% weniger Patienten die Einschlusskriterien für eine Chemotherapie erfüllen. Selbst bei einem 
Cut-off-Wert von $45 \mathrm{ml} / \mathrm{min}$ wären ca. $25 \%$ weniger Patienten für eine Chemotherapie geeignet. Der Altersgipfel der Inzidenz des UTUC liegt außerdem bei 70-90 Jahren [1], also in einer Gruppe, die besonders stark vom postoperativen GFR-Abfall betroffen ist und daher häufig Kontraindikationen sowohl gegen die prä- als auch die postoperative Chemotherapie darstellt [22].

In der Subgruppenanalyse war ein signifikanter Effekt nur bei Patienten der Gemcitabin-Cisplatin-Gruppe nachweisbar. Es bleibt also fraglich, ob Patienten mit stark eingeschränkter Nierenfunktion von der carboplatinbasierten Chemotherapie profitieren. Man kann also für diese Subgruppe eigentlich keine Empfehlung zu einer adjuvanten Chemotherapie aussprechen, da ein Vorteil für das progressionsfreie Überleben in dieser speziellen Gruppe zunächst erst einmal belegt werden müsste. Dies ist ein weiteres Argument, die Wirksamkeit einer neoadjuvanten Therapie beim UTUC genauer zu erforschen. Um die Durchführbarkeit eines solchen neoadjuvanten Ansatzes zu prüfen, begannen die Autoren 2018 diesbezüglich eine neue randomisierte Studie (URANUS/NCT02969083). Sie schließt auch mit MVAC (Methotrexat, Vinblastin, Doxorubicin und Cisplatin) eine weitere Chemotherapeutikakombination ein, die beim Blasenkarzinom und beim metastasierten UTUC in manchen Studien ein deutlich verbessertes Gesamtüberleben im Vergleich zur Kombination Gemcitabin/Cisplatin zeigte [23, 24].

Und zuletzt konnte wegen der vorzeitigen Veröffentlichung wesentlicher Daten der POUT-Studie der Effekt der adjuvanten Chemotherapie auf das Gesamtüberleben leider noch nicht evaluiert werden. Die Nachbeobachtungszeit war dazu zu kurz. Damit wurde ein entscheidender Endpunkt der Studie zur Beurteilung der therapeutischen Effizienz verspielt. Aus anderen Studien zum UTUC ist jedoch bekannt, dass signifikante Unterschiede im krankheitsfreiem Überleben auch zu Verbesserungen im Gesamtüberleben führen [25]. Unter Einschränkungen kann damit das krankheitsfreie Überleben als sinnvoller Surrogatparameter für das UTUC eingesetzt werden, auch für die zukünftige klinische Studie. Mit diesem Trick lässt sich aus der signifikanten Verbesserung des krankheitsfreien Überlebens in der POUT-Studie auch auf eine Verbesserung des Gesamtüberlebens dieser Patientengruppe schließen.

\section{Fazit}

Es ist sinnvoll, UTUC als eigenständiges Krankheitsbild vom Urothelkarzinom der Blase abzugrenzen und dementsprechend zu behandeln.

Die Forderung der Autoren, das adjuvante Therapieschema mit Gemcitabin/Cis- oder Carboplatin als neuen Goldstandard in der Behandlung des fortgeschrittenen UTUC anzusehen, ist berechtigt, und die Daten sollten bei zukünftigen Empfehlungen und Leitlinien berücksichtigt werden.

Es besteht noch Forschungs- und Optimierungsbedarf bezüglich der Patientenselektion und des optimalen Therapieregimes. In der Gruppe ohne Lymphknotenbefall zeigte die adjuvante Chemotherapie nämlich einen Nutzen, weshalb es fraglich ist, ob die erweiterte Lymphknotendissektion noch einen zusätzlichen Vorteil bringen würde.

Zusätzlich bleibt zu klären, ob ein neoadjuvanter Ansatz (wie beim Blasenkarzinom) nicht auch beim UTUC eingesetzt werden sollte, insbesondere da bei diesem Ansatz die operationsbedingte Reduktion der Nierenfunktion noch nicht einschränkend bei der Durchführung einer volldosierten Chemotherapie ins Gewicht fällt.

\section{Lea Pape, Johanna Richter und Jürgen Dunst, Kiel}

Funding Open Access funding provided by Projekt DEAL.

Interessenkonflikt L. Pape, J. Richter und J. Dunst geben an, dass kein Interessenkonflikt besteht.

Open Access Dieser Artikel wird unter der Creative Commons Namensnennung 4.0 International Lizenz veröffentlicht, welche die Nutzung, Vervielfältigung, Bearbeitung, Verbreitung und Wiedergabe in jeglichem Medium und Format erlaubt, sofern Sie den/die ursprünglichen Autor(en) und die Quelle ordnungsgemäß nennen, einen Link zur Creative Commons Lizenz beifügen und angeben, ob Änderungen vorgenommen wurden.

Die in diesem Artikel enthaltenen Bilder und sonstiges Drittmaterial unterliegen ebenfalls der genannten Creative Commons Lizenz, sofern sich aus der Abbildungslegende nichts anderes ergibt. Sofern das betreffende Material nicht unter der genannten Creative Commons Lizenz steht und die betreffende Handlung nicht nach gesetzlichen Vorschriften erlaubt ist, ist für die oben aufgeführten Weiterverwendungen des Materials die Einwilligung des jeweiligen Rechteinhabers einzuholen.

Weitere Details zur Lizenz entnehmen Sie bitte der Lizenzinformation auf http://creativecommons.org/licenses/by/4.0/deed.de.

\section{Literatur}

1. Rouprêt M, Babjuk M, Compérat E, Zigeuner R, Sylvester RJ, Burger M et al (2018) European Association of Urology Guidelines on Upper Urinary Tract Urothelial Carcinoma: 2017 Update. Eur Urol 73(1):111-122 (http://www.ncbi.nlm.nih.gov/pubmed/28867446)

2. Soria F, Shariat SF, Lerner SP, Fritsche HM, Rink M, Kassouf W et al (2017) Epidemiology, diagnosis, preoperative evaluation and prognostic assessment of upper-tract urothelial carcinoma (UTUC). World J Urol 35(3):379-387

3. Audenet F, Isharwal S, Cha EK, Donoghue MTA, Drill EN, Ostrovnaya I et al (2019) Clonal relatedness and mutational differences between upper tract and bladder urothelial carcinoma. Clin Cancer Res 25(3):967-976 (http://www.ncbi.nlm.nih.gov/pubmed/ 30352907)

4. Winters BR, De Sarkar N, Arora S, Bolouri H, Jana S, Vakar-Lopez F et al (2019) Genomic distinctions between metastatic lower and upper tract urothelial carcinoma revealed through rapid autopsy. JCI Insight. https://doi.org/10.1172/jci.insight.128728 (http:// www.ncbi.nlm.nih.gov/pubmed/31145100) 
5. Green DA, Rink M, Xylinas E, Matin SF, Stenzl A, Roupret M et al (2013) Urothelial carcinoma of the bladder and the upper tract: Disparate twins. J Urol 189:1214-1221 (http://www.ncbi.nlm.nih. gov/pubmed/23023150)

6. Catto JWF, Yates DR, Rehman I, Azzouzi AR, Patterson J, Sibony $\mathrm{M}$ et al (2007) Behavior of urothelial carcinoma with respect to anatomical location. J Urol 177(5):1715-1720 (http://www.ncbi. nlm.nih.gov/pubmed/17437794)

7. Audenet F, Yates DR, Cussenot O, Rouprêt M (2013) The role of chemotherapy in the treatment of urothelial cell carcinoma of the upper urinary tract (UUT-UCC). Urol Oncol 31:407-413 (http:// www.ncbi.nlm.nih.gov/pubmed/20884249)

8. Leow JJ, Martin-Doyle W, Fay AP, Choueiri TK, Chang SL, Bellmunt J (2014) A systematic review and meta-analysis of adjuvant and neoadjuvant chemotherapy for upper tract urothelial carcinoma. Eur Urol 66:529-541 (http://www.ncbi.nlm.nih.gov/pubmed/ 24680361)

9. Goldberg H, Klaassen Z, Chandrasekar T, Sayyid R, Kulkarni GS, Hamilton RJ et al (2018) Does perioperative chemotherapy improve survival in upper tract urothelial carcinoma? A population based analysis. Oncotarget 9(27):18797-18810

10. Seisen T, Jindal T, Karabon P, Sood A, Bellmunt J, Rouprêt M et al (2017) Efficacy of systemic chemotherapy plus radical nephroureterectomy for metastatic upper tract urothelial carcinoma. Eur Urol 71(5):714-718 (http://www.ncbi.nlm.nih.gov/pubmed/27912971)

11. Necchi A, Lo Vullo S, Mariani L, Moschini M, Hendricksen K, Rink M et al (2018) Adjuvant chemotherapy after radical nephroureterectomy does not improve survival in patients with upper tract urothelial carcinoma: a joint study by the European Association of Urology-Young Academic Urologists and the Upper Tract Urothelial Carcinoma Collaboration. BJU Int 121(2):252-259 (http:// www.ncbi.nlm.nih.gov/pubmed/28940605)

12. Kim DK, Lee JY, Kim JW, Hah YS, Cho KS (2019) Effect of neoadjuvant chemotherapy on locally advanced upper tract urothelial carcinoma: a systematic review and meta-analysis. Crit Rev Oncol Hematol 135:59-65 (http://www.ncbi.nlm.nih.gov/pubmed/ 30819447)

13. Gregg RW, Vera-Badillo FE, Booth CM, Mahmud A, Brundage M, Leveridge MJ et al (2018) Perioperative chemotherapy for urothelial carcinoma of the upper urinary tract: a systematic review and meta-analysis. Crit Rev Oncol Hematol 128:58-64 (http://www.ncbi. nlm.nih.gov/pubmed/29958631)

14. Fujita K, Taneishi K, Inamoto T, Ishizuya Y, Takada S, Tsujihata M et al (2017) Adjuvant chemotherapy improves survival of patients with high-risk upper urinary tract urothelial carcinoma: a propensity score-matched analysis. BMC Urol 17(1):110

15. Hellenthal NJ, Shariat SF, Margulis V, Karakiewicz PI, Roscigno M, Bolenz $\mathrm{C}$ et al (2009) Adjuvant chemotherapy for high risk upper tract urothelial carcinoma: results from the upper tract urothelial carcinoma collaboration. J Urol 182(3):900-906
16. Matin SF, Margulis V, Kamat A, Wood CG, Grossman HB, Brown GA et al (2010) Incidence of downstaging and complete remission after neoadjuvant chemotherapy for high-risk upper tract transitional cell carcinoma. Cancer 116(13):3127-3134

17. Quhal F, Mori K, Sari Motlagh R, Laukhtina E, Pradere B, Rouprêt $\mathrm{M}$ et al (2020) Efficacy of neoadjuvant and adjuvant chemotherapy for localized and locally advanced upper tract urothelial carcinoma: a systematic review and meta-analysis. Int $\mathrm{J}$ Clin Oncol. https://doi.org/10.1007/s10147-020-01650-9 (http://www. ncbi.nlm.nih.gov/pubmed/32206939)

18. Hosogoe S, Hatakeyama S, Kusaka A, Hamano I, Iwamura H, Fujita $\mathrm{N}$ et al (2018) Platinum-based neoadjuvant chemotherapy improves oncological outcomes in patients with locally advanced upper tract urothelial carcinoma. Eur Urol Focus 4(6):946-953 (http:// www.ncbi.nlm.nih.gov/pubmed/28753881)

19. Crabb SJ (2020) Treatment of upper urinary tract urothelial carcinoma. Lancet 395(10232):1232-1234. https://doi.org/10.1016/ S0140-6736(20)30519-5

20. Raghavan D, Bawtinhimer A, Mahoney J, Eckrich S, Riggs S (2014) Adjuvant chemotherapy for bladder cancer-why does level 1 evidence not support it? Ann Oncol 25(10):1930-1934 (http:// www.ncbi.nlm.nih.gov/pubmed/24569916)

21. Chitale S, Mbakada R, Irving S, Burgess N (2008) Nephroureterectomy for transitional cell carcinoma-The value of pre-operative histology. Ann R Coll Surg Engl 90(1):45-50 (http://www.ncbi. nlm.nih.gov/pubmed/18201500)

22. Kaag MG, O’Malley RL, O’Malley P, Godoy G, Chen M, Smaldone MC et al (2010) Changes in renal function following nephroureterectomy may affect the use of perioperative chemotherapy. Eur Urol 58(4):581-587 (http://www.ncbi.nlm.nih.gov/pubmed/ 20619530)

23. Zargar H, Shah JB, van Rhijn BW, Daneshmand S, Bivalacqua TJ, Spiess PE et al (2018) Neoadjuvant dose dense MVAC versus gemcitabine and cisplatin in patients with cT3-4aN0M0 bladder cancer treated with radical cystectomy. J Urol 199(6):1452-1458 (http:// www.ncbi.nlm.nih.gov/pubmed/29329894)

24. Hsieh MC, Chiang PH, Rau KM, Chen YY, Su YL, Huang CH (2015) The comparison of oncologic outcomes between metastatic upper tract urothelial carcinoma and urothelial carcinoma of the bladder after cisplatin-based chemotherapy. Urol Oncol 33(11):495.e9-495.e14 (http://www.ncbi.nlm.nih.gov/pubmed/ 26254698)

25. Fajkovic H, Cha EK, Xylinas E, Rink M, Pycha A, Seitz C et al (2013) Disease-free survival as a surrogate for overall survival in upper tract urothelial carcinoma. World J Urol 31(1):5-11 (http:// www.ncbi.nlm.nih.gov/pubmed/23011256) 